\title{
UNKNOTTING NUMBER ONE KNOTS ARE PRIME: A NEW PROOF
}

\author{
XINGRU ZHANG
}

(Communicated by Frederick R. Cohen)

\begin{abstract}
An alternative proof for unknotting number one knots being prime is given.
\end{abstract}

The unknotting number of a knot $K \subset S^{3}$, denoted by $u(K)$, is the minimum number of crossing changes required to unknot $K$. Obviously $u(K)$ is a knot invariant, but surprizingly little is known about it. The following theorem of M. Scharlemann proves a long standing conjecture.

Theorem [S, Theorem]. A knot $K \subset S^{3}$ with $u(K)=1$ is prime.

To prove the above theorem, Scharlemann developed certain combinatorics dealing with planar graphs coming from an intersection of two special planar surfaces. Later in [ST], Scharlemann and Thompson gave another proof of the theorem [ST, Corollary 3.4], that is based on a delicate application of the sutured manifold structure theory. In this note we point out a new proof, applying only some existing results. In fact the proof follows immediately from the following three known lemmas.

Lemma 1 [L, Lemma 1]. Let $K$ be a knot in $S^{3}$ with $u(K)=1$, and let $M_{K}$ be the double cover of $S^{3}$ branched over $K$. Then $M_{K}$ can be obtained by $n / 2$-surgery on some knot in $S^{3}, n$ being an odd integer.

Lemma 2 [GL, Theorem 1]. Let $K$ be a knot in $S^{3}$, and let $K(m / l)$ denote the manifold obtained by $m / l$-surgery on $K$. Then $K(m / l)$ is a prime manifold if $|l| \neq 1$.

Lemma 3 [KT, Corollary 4]. Let $K$ be a knot in $S^{3}$. Then the double cover $M_{K}$ of $S^{3}$ branched over $K$ is a prime manifold iff $K$ is a prime knot.

Proof of Theorem. Since $u(K)=1, M_{K}$ is a prime manifold by Lemmas 1 and 2. Hence $K$ is a prime knot by Lemma 3.

The author has learned that a similar approach was pointed out by C. Gordon in a lecture given at Santa Barbara.

Received by the editors June 5, 1990 .

1980 Mathematics Subject Classification (1985 Revision). Primary 57M25. 


\section{REFERENCES}

[GL] C. McA. Gordon and J. Luecke, Only integral Dehn surgery can yield reducible manifolds, Math. Proc. Cambridge Philos. Soc. 102 (1987), 97-101.

[KT] P. K. Kim and J. L. Tollefson, Splitting the PL involutions of nonprime 3-manifolds, Michigan Math. J. 27 (1980), 259-274.

[L] W. B. R. Lickorish, The unknotting number of a classical knot, Contemp. Math., vol. 44, 1985, pp. 117-121.

[S] M. G. Scharlemann, Unknotting number one knots are prime, Invent. Math. 82 (1985), 37-55.

[ST] M. G. Scharlemann and A. Thompson, Unknotting number, genus, and companion tori, Math. Ann. 280 (1988), 191-205.

Mathematics Department, University of British Columbia, Vancouver, British Columbia, CANada, V6T 1Y4 